\title{
Surrounding matter theory
}

\author{
Frederic Lassiaille, ${ }^{1, *}$ \\ ${ }^{1}$ FL research, France
}

\begin{abstract}
S.M.T. (Surrounding Matter Theory), an alternative theory to dark matter, is presented. It is based on a modification of Newton's law. This modification is done by multiplying a Newtonian potential by a given factor, which is varying with local distribution of matter, at the location where the gravitational force is exerted. With this new equation the model emphasizes that a gravitational force is roughly inversely proportional to mass density at the location where this force is applied. After presentation of the model, its dynamic is quickly applied to cosmology and galaxy structure. Some possible caveats of the model are identified. But the simple mechanism described above suggests the idea of a straightforward solution to the following issues: virial theorem mystery, the bullet cluster (" $1 \mathrm{E}$ 0657-56" galaxy clusters) issue, the strong relative velocity of its subclusters, the value of cosmological critical density, the fine tuning issue, and expansion acceleration. Nucleosynthesis is not explained and would require a different model for radiation era. But a de Sitter Universe is predicted, this means that the spatial curvature, $K$, is 0 , and today's deceleration parameter, $q$, is -1 . The predicted time since last scattering is $68 \mathrm{~h}^{-1}$ Gyr. With this value SMT explains heterogeneities of large scale structure and galaxy formation. Each kind of experimental speed profiles are retrieved by a simulation of a virtual galaxy. In the simulations, ring galaxies are generated by SMT dynamic itself, without the help of any particular external event. Those studies give motivation for scientific comparisons with experimental data.
\end{abstract}

\section{Introduction}

This article presents Surrounding Matter Theory (SMT), and is a very quick survey of its predictions and results. This model is an alternative to dark matter in solving today's gravitational mysteries. The solving principle is a modification of Newton's law. SMT is composed of 1 equation and 2 parameters. This simplicity allows a robust survey of the model, and restricts enormously the amount of possible regression on other parts of physics. Stated in one sentence, the whole behavior of those equations is that a gravitational force is inversely proportional to matter density at the location where the force is exerted.

The first motivation is an old one: Mach's principle [1]. Here an attempt is made to express fully this principle by getting the ratio of inertial to gravitational mass, or let's say a "modified $G$ ", directly coupled to matter. And to avoid any resulting changes in the local behaviour of matter, and the local equations of motion, the first idea is to restrict this $G$

*E-mail: frederic.lassiaille@yahoo.com 
variation to large distances only. The second idea is a novelty: relating this variation only to matter located at the location where the force is exerted. This will keep valid linearity with attracting matter. The second motivation concerns General Relativity (GR). Indeed, in GR, Bianchi identity and the resulting null covariant divergence $\nabla^{\mu} G_{\mu v}=0$ of $G_{\mu v}$ Einstein tensor is linked directly to energy conservation $\nabla^{\mu} T_{\mu v}=0$ of $T_{\mu v}$, the stress-energy tensor, via Einstein equation. But one could notice that the first one comes from pure geometry, whereas the second one comes from physics, namely energy conservation physics principle. This leads me to consider the possibility that those 2 equations are not directly binded together, but could rely on one another through a more complex relation. In particular, I allowed for the Einstein tensor not to be proportional to the stress-energy tensor but rather to be a more general function of it. Furthermore, for reasons such as linearity with respect to energy, I was led to the form $G_{\mu \nu}=\kappa C_{\mu}^{\rho} C_{v}^{\sigma} T_{\rho \sigma} . \kappa$ is the multiplicative constant of Einstein equation $G_{\mu v}=\kappa T_{\mu v} . C_{\mu}^{v}$ is a mixed tensor which remains to be calculated using the GR case and the non relativistic limit. For the latter this modification undergo to the simple modification of a gravitational potential. This led to $\Phi=C_{S M T} \Phi_{n}$, where $\Phi$ is the final gravitational potential. $\Phi_{n}$ is a Newtonian potential. $C_{S M T}$ is a varying factor, being a function of matter density at the location where the force is exerted. Today's gravitational mysteries are solved or partially solved using various different theories, for example in [2-8]. After the SMT description, its dynamic will be illustrated in the context of the appearance of those mysteries.

\section{The model}

As introduced above, the starting point is the following gravitational potential equation.

$$
\Phi_{n}=-\frac{M G}{x}
$$

$x$ is the distance from an attracting infinitesimal object, $M$ the mass of this object and $G$ is gravitational constant. The model consists of modifying this equation. Three more variables are added.

The first one is $\rho$, mass density calculated in a sphere of ray $r_{\max }$ around the location where the force is applied. This sphere will be called the "SMT sphere" in this document. There is $r_{\max }=10 \mathrm{~h}^{-1} \mathrm{kpc}, \mathrm{h}$ being Hubble constant in units of $100 \mathrm{~km} \mathrm{~s}^{-1} \mathrm{Mpc}^{-1}$. Using $H_{0}=67 \mathrm{~km} \mathrm{~s}^{-1} \mathrm{Mpc}^{-1}$, there is $r_{\max } \cong 15 \mathrm{kpc}$. It is this $r_{\max }$ value which will be used in this document. $\rho_{0}$ is today's value of $\rho$ in the vicinity of the sun. It will be used: $\rho_{0}=0.9810^{-21} \mathrm{~kg} / \mathrm{m}^{3}$. The modified potential equation is the following.

$$
\Phi=-\frac{M G}{x} \underbrace{\frac{\alpha_{0} \rho_{0}+\rho_{u 0}}{\alpha \rho+\rho_{u}}}_{C_{S M T}}
$$


The second variable is $\rho_{u}$, the Universe mass density. $\rho_{u 0}$ is today's value of $\rho_{u}$. The third variable is $\alpha$, which can be set to 2 values only. There is $\alpha=\alpha_{0}=1.610^{-5}$ inside the galaxies, and $\alpha=1$ outside any galaxy. Those values are stated to be independent of Universe expansion.

\section{Relativistic version}

In the equation giving $C_{S M T}$, through a Lorentz transform, each parameter on the numerator evolves exactly the same way as its corresponding counterpart in the denominator. The result is that $C_{S M T}$ is a Lorentz invariant.

The first remark before searching for a relativistic version is the role of $M$ in equation (1) and (2). Since $C_{S M T}$ depends only on matter at the location where the force is exerted, it does not depend directly on $M$. Therefore like equation (1), equation (2) shows acceleration as being linear with respect to attracting matter $(M)$. This is a distinctive characteristic of SMT as a modification of Newton's law. Only variations with distance ( $x$ ), and $G$ (in some sense, because it is in fact $C_{S M T} G$ ) are modified, not variation with $M$. One could even guess that this characteristic would hold with the relativistic version of SMT.

Now modifying Einstein equation with a metric related scalar would not give back equation (2) as the non relativistic limit. It would be the same with any scalar-tensor theory [9], which would finally add a scalar tensor to the physical stress-energy tensor. Einstein modified equation would not show its left-handed term as being strictly linear with respect to attracting energy. Any modification acting on Lagrangian level would probably result in the same caveats, except if modifying the scalar curvature itself in GR Lagrangian. SMT Lagrangian will be given below, but only after calculation of the modified Einstein equation. For this calculation the algebraic constraints are the following.

- Bianchi identity,

- stress-energy tensor conservation,

- variation of $C_{S M T}$,

- linearity of curvature with respect to attracting matter.

The latter implies that any added term is forbidden. Therefore a simple solution is to replace $C_{S M T}$ by its space-time tensorial expression. $C_{S M T}$ is replaced by $C_{\mu}^{\rho} C_{v}^{\sigma}$, where $C_{v}^{\mu}$ is a mixed multiplying tensor, allowing a different factor than $C_{S M T}$ to be applied to the space components of $T_{\mu \nu}$. Since the result must retrieve equation (2) in the nonrelativistic case, there is $C_{0}^{0}{ }^{2}=C_{S M T}$ in the co-moving bases. Bianchi identity and energy conservation along with $C_{S M T}$ variation imply a separate variation of each $C_{\mu}^{v}$ factor in front of its corresponding component in the stress-energy tensor. Now this factor depends on the component being multiplied, that is, it depends on $\mu$ and $v$. These constraints lead to the generalization of $R_{\mu v}-\frac{1}{2} R g_{\mu v}=\frac{8 \pi G}{c^{4}} T_{\mu v}$ by the following group of equations. 


$$
\begin{aligned}
& R_{\mu \nu}=\frac{1}{2} R g_{\mu v}=\frac{8 \pi G}{c^{4}} S_{\mu v} \\
& S_{\mu v}=C_{\mu}^{\rho} C_{v}^{\sigma} T_{\rho \sigma} \\
& C_{0}^{v}=\sqrt{C_{S M T}} \delta_{0}^{v} \quad C_{i}^{v}=\sqrt{s} \delta_{i}^{v}
\end{aligned}
$$

$c$ is the speed of light, $R_{\mu \nu}$ is the Ricci tensor, $g_{\mu \nu}$ is the metric, $R$ is the trace of $R_{\mu \nu}$. $\delta_{\mu}^{v}$ are Kronecker symbols and $i$ indice is varying between 1 and 3. Equations (3) shows that $C_{\mu}^{v}$ is a time dilation by the $\sqrt{C_{S M T}}$ factor, and a space dilation by the $\sqrt{s}$ factor, $s$ being a positive scalar. For calculating $s, \nabla^{\mu} G_{\mu \nu}=0$ implies the following.

$$
\begin{aligned}
& 2 \partial_{0}\left(C_{S M T} \rho\right)+\left(C_{S M T} \rho+s T_{i}^{i}\right) g^{i i} \partial_{0} g_{i i}=0 \\
& \begin{aligned}
2 \partial_{i}\left(s T_{i}^{i}\right)+\left(C_{S M T} \rho\right. & \left.+s T_{i}^{i}\right) g^{00} \partial_{i} g_{00} \\
& +s\left(T_{i}^{i}-T_{k}^{k}\right) g^{k k} \partial_{i} g_{k k}=0
\end{aligned}
\end{aligned}
$$

Here it has been supposed $c=1$ for simplification. The notation $\partial_{\mu}=\partial / \partial x^{\mu}$ has been used. The calculation is done in co-moving bases such as $g_{\mu \nu}$ matrices are diagonal, and supposing no shear forces in $T_{\mu \nu}$. Therefore $T_{\mu \nu}$ matrices are also diagonal. Here the non SMT case $C_{S M T}=1$ is simply solved by setting $s=1$. In the general case equations (4) allow a calculation of a finite $s$, but only under the supposition of a non null pressure $T_{i}^{i} \neq 0$. Otherwise it corresponds to the more general hypothesis of a null stress tensor. And this can be argued as being never completely physically relevant. A static Universe is also forbidden for calculating $s$ (exactly there must be $\partial_{0} g_{i i} \neq 0$ ). And it can be argued also that a static Universe is never physically relevant.

Nevertheless, for avoiding those slight caveats, another solution is the following. As mentioned in the motivation, let's postulate that the null covariant divergence $\nabla^{\mu} G_{\mu \nu}=0$ of Einstein tensor is independent of energy conservation $\nabla^{\mu} T_{\mu \nu}=0$, in the general case. This can be modeled by a $S_{\mu \nu}$ isotropic space part, independent of $T_{\mu \nu}$. In this case $\nabla^{\mu} S_{\mu \nu}=0$ yields the following, using again the co-moving bases and searching for a diagonal $S_{\mu \nu}$ matrix, but now without any supposition on $T_{\mu \nu}$.

$$
\begin{aligned}
& 2 \partial_{0}\left(C_{S M T} \rho\right)+\left(C_{S M T} \rho+P_{S M T}\right) g^{i i} \partial_{0} g_{i i}=0 \\
& 2 \partial_{i} P_{S M T}+\left(C_{S M T} \rho+P_{S M T}\right) g^{00} \partial_{i} g_{00}=0
\end{aligned}
$$


It has been written $P_{S M T}=S_{11}=S_{22}=S_{33}$. This should allow to calculate $P_{S M T}$ in any cases. But here the non SMT case $C_{S M T}=1$ implies either an unrealistic simplification of the physical stress tensor, or its independence from space-time curvature. Therefore validation of GR equation in the particular context of a non null space part of the stressenergy tensor must be searched for, in order to possibly invalidate this last solution, and then choose the other one. This completes the construction of equations (3). Finally, those equations must be validated backward. And the result is that they fulfill each of their initial constraints. In the specific case of today's solar system, SMT prediction is exactly GR. More generally, GR is retrieved in the "constant $C_{S M T}=1$ " case. This is of course mandatory. Equation (2) is retrieved in the non-relativistic case. But in the other cases, differences with GR must be analyzed.

\section{Possible regressions}

In the "constant $C_{S M T}$ " case, GR is not exactly retrieved: if $C_{S M T} \neq 1$, there is also $S \neq 1$, with $s \neq C_{S M T}$. Therefore, not only $G$ appears to be different, but also a dilatation factor appears on the space part of the stress-energy tensor. This implies that some PPN formalism parameters will be different from their GR values. But comparing those new predicted values with reality would require testing gravity today $15 \mathrm{kpc}$ beyond the solar system, or inside the solar system but more than 50000 years in the past (since there is $15 \mathrm{kpc} \cong 50000 \mathrm{LY}$ ). At first glance those experiments seems difficult to realize.

Even the "varying $C_{S M T}$ " case in which matter density is varying consistently, must be thoroughly analyzed. In particular, a possible time variation of $C_{S M T}$ in the solar system must be studied. The resulting apparent $G$ variation must be calculated from matter density variation in the SMT sphere around the sun, and then compared to experimental data.

The case of binary stars and exoplanets will be addressed further in this document.

An important case is the spherically symmetric Universe. The Schwarzschild metric behaves like the classical one but with a different $G$ value. Here emptiness $T_{\mu \nu}=0$ leads to a radically unrealistic situation: there is a singularity everywhere in the Universe. And this is, now, compatible with Mach's principle. The cosmological case will be addressed below.

\section{Lagrangian version}

Let's review GR Lagrangian: $L_{G R}=\int \sqrt{-g} R d x^{4}+L_{M} \cdot g$ is the metric determinant and $L_{M}$ the energy Lagrangian such as $T_{\mu v}=-\frac{1}{8 \pi G} \frac{\delta L_{M}}{\delta g^{\mu \nu}}$. Now let's calculate $L_{S M T}$, the SMT Lagrangian replacing $L_{G R}$. Writing $D_{v}^{\mu}$ such as $D_{\lambda}^{\mu} C_{v}^{\lambda}=C_{\lambda}^{\mu} D_{v}^{\lambda}=\delta_{v}^{\mu}$, 
$R_{S M T}=g^{\mu \nu} D_{\mu}^{\rho} D_{v}^{\sigma} R_{\rho \sigma}$, and $L_{C S M T}$ such as $L_{C S M T}=-\int \sqrt{-g} X d x^{4}, X$ being a scalar such as $\frac{\delta X}{\delta g^{\mu \nu}}=g^{\rho \sigma} R_{\lambda \delta} \frac{\delta\left(D_{\rho}^{\lambda} D_{\sigma}^{\delta}\right)}{\delta g^{\mu \nu}}$, there is:

$$
L_{S M T}=\int \sqrt{-g} R_{S M T} d x^{4}+L_{M}+L_{C S M T}
$$

It looks like GR Lagrangian. $R$ has been replaced by $R_{S M T}$, which can be interpreted as $R$ modified by $C_{S M T}$. An added term, $L_{C S M T}$, has appeared. It can be interpreted as the Lagrangian corresponding to $C_{S M T}$. The following suppositions have been done in order to yield equation (6).

- The mean value of $C_{S M T}$ has been supposed constant over the Universe, this "mean" value being calculated over a given distance greater than the visible Universe size.

- $C_{S M T}$ is supposed to vary around this mean value regularly (that is, with a frequency bounded by a minimum value)

\section{Gravitational mysteries}

\subsection{Aim of these overviews}

Some gravitational mysteries will be studied in this document. This will be done in a very quick, mostly qualitative, and carefull manner. These studies are not scientific comparisons. They are only very quick applications of SMT to some particular contexts. Their aim is only to reveal some interesting characteristics of SMT dynamic.

\subsection{Critical Universe density}

In the context of Friedmann-Lemaitre-Robertson-Walker (FLRW) metric, there is $\rho=\rho_{u}$. This is imposed by Universe homogeneity in this case. First of all, let's calculate the first Friedmann-Lemaître (FL) equation.

$$
H^{2}+\frac{K c^{2}}{a^{2}}=\frac{8 \pi G}{3} C_{S M T} \rho_{u}
$$

This result is independent of the choice of the model, that is, the choice between equations (4) or equations (5). $H$ is Hubble parameter, $a$ is the scale factor, and $K$ is space curvature. In FLRW metric context, there is $\alpha=1$ therefore equation (2) shows that $C_{S M T} \rho_{u}$ is constant. This will produce dramatic simplifications of cosmological model. Indeed, writing $P_{S M T}=w_{S M T} C_{S M T} \rho$, the classical version of energy conservation under FLRW metric implies $w_{S M T}=-c^{2}$ and $K=0$ : FL equations yield a de Sitter Universe. And once again, this result is independent of the choice of the model, that is, the choice 
between equations (4) and equations (5). Let's notice that another possible solution from any chosen group of equations, (4) or (5), could be a static Universe with a positive space curvature. But this is physically irrelevant. The result is that $w_{S M T}$ has no interesting physical meaning. In FLRW co-moving bases $S_{\mu v}$ is simply $-\rho_{c} c^{2}$ times the Minkowski metric diagonal matrix $\eta_{\mu v}$, such as $\eta_{0 v}=-\delta_{0 v}$ and $\eta_{i v}=\delta_{i v}$ for $v$ between 0 and 3 . Because of the "well-suited" tensor product of equations (3), the physically meaning state equation $\rho=w P, P$ being $T_{\mu \nu}$ pressure, has no specific effect on space-time curvature. Everything acts as if $T_{\mu v}$ has been replaced by $S_{\mu v}$, having a constant matrix in FLRW co-moving bases. Now, equation (7) can be written:

$$
H^{2}=\frac{8 \pi G}{3} \rho_{c}
$$

This equation is valid from last scattering until today. Before last scattering, SMT is no longer valid. The solution of this de Sitter universe is the following.

$$
a=a_{0} e^{H_{0} t}
$$

It will be supposed $a=a_{0}=1$ at today's time. The predicted elapsed time since last scattering, $T_{L S}$, is given by the following equation, using $a_{l s}=1 /\left(1+z_{l s}\right)$.

$$
T_{L S}=\frac{\ln \left(a_{l s}^{-1}\right)}{H_{0}}=68 \mathrm{~h}^{-1} \mathrm{Gyr}
$$

This is in strong disagreement with $\Lambda$ CDM model value of $13.798 \pm 0.037 \mathrm{Gyr}$ $9.35 \mathrm{~h}^{-1} \mathrm{Gyr}$ (using $H_{0}=67.80 \mathrm{~km} \mathrm{~s}^{-1} \mathrm{Mpc}^{-1}$ ). It could be allowed by a much longer dark age period. But such a duration explains the formation of galaxies. For example, now a galaxy such as UGC 2885 [10] will have more than $(68 / 9.35) \times 12 \cong 87$ revolutions to create, since last scattering, in place of only 12 revolutions with $\Lambda \mathrm{CDM}$ value. Also, the localization of UDFJ-39546284 [11], [12] at $z \cong 12$ is possible in the context of SMT.

The important result of this chapter is that the issue of critical Universe density [13], [14], is solved directly and in a simple manner by SMT. No more cosmological constant is needed.

\subsection{Nucleosynthesis, fine tuning, singularity, particle's horizon and acceleration of Universe's expansion.}

In the context of SMT, there is no fine tuning issue, since matter density has been simplified during the modification of FL equations. At first glance, particle's horizon issue is solved by the disappearance of any time limit in the past, ensuring the Universe's 
homogeneity and isotropy, and "big-bang" singularity is solved altogether. These are direct consequences of the previous calculations.

But primordial nucleosynthesis is not explained by SMT: the predicted Deuterium abundances are incorrect. It would probably require microscopic scale, or high energy specific predictions for studying radiation-dominated era. And this is a domain in which SMT is probably inoperative. Therefore particle's horizon and "big-bang" singularity would need different or refined explanations.

Let's write the deceleration parameter $q$ such as $q=-\frac{\ddot{a} a}{\dot{a}^{2}}, \dot{a}=\frac{d a}{d t}, \ddot{a}=\frac{d^{2} a}{d t^{2}}$. From equation (8), there is:

$$
q=-1
$$

This is in accordance with experimental data [15], Table 8. SMT predictions, $K=0$ and equation (11), are compatible with today's measured values, [15], [16].

\subsection{Heterogeneity of large scale structure}

The problem of heterogeneities of large scale structure [17] can first be addressed with Jeans instability. Let's start from the classical collapse time $t_{j}$, valid under Newton's law.

$$
t_{j}=\frac{1}{\sqrt{G \rho_{u}}}
$$

This value in the context of SMT is also calculated from hydrodynamic and is the following.

$$
t_{j}^{\prime}=\frac{1}{\sqrt{G \rho_{c}}}=28 \mathrm{~h}^{-1} \mathrm{Gyr}
$$

Equations (12) and (13) are valid in a homogenous Universe, at any time. But equation (13) shows a very important difference: SMT collapse calculation is no longer driven by Universe's expansion, like Newton's law collapses are. Using $c_{s}=5 \mathrm{~km} / \mathrm{s}$ for the sound speed just after decoupling, Jeans length is the following.

$$
l_{j}{ }^{\prime}=c_{s} t_{j}{ }^{\prime}=140 h^{-1} \mathrm{kpc}
$$

This allows for the creation of voids and walls structures.

Now let's suppose a wall, located between $x=0$ and $x=x_{\text {wall }}>0$, parallel to the $y-z$ plane, at today's time. From equation (2), if $\vec{a}$ is the SMT acceleration corresponding to any $\vec{a}_{n}$ Newtonian acceleration, there is: 


$$
\vec{a}=\frac{2 \rho_{c}}{\rho_{u 0}+\rho}\left(1+\frac{r}{\rho_{u 0}+\rho} \frac{\partial \rho}{\partial r}\right) \vec{a}_{n}
$$

$r$ is the distance between the infinitesimal object generating $\vec{a}_{n}$, and the location where it is exerted. The distance between two walls is always far greater than Jeans length given by equation (14). Therefore any hydrodynamic equilibrium will be driven by equation (15) only. The astonishing prediction is that no more counteracting pressure is required in order to achieve a hydrodynamic equilibrium. And this is even independent of the exact wall and filament structure. Between the filaments and walls, if one neglect the matter density with respect of matter density of the wall and filaments, there exist a completely new, stable equilibrium, given by the following equation. It expresses the distribution of matter density, valid, for example, on the right hand side of this wall.

$$
\rho=\left(\rho_{\text {wall }}+\rho_{u 0}\right) \frac{x_{\text {wall }}}{x}-\rho_{u 0}
$$

$\rho_{\text {wall }}$ is the matter density of the wall. The approximations driving this equation were $x>>x_{\text {wall }}$, and only small perturbations allowed with $\rho<<\rho_{\text {wall }}$. Supposing also $\rho_{\text {wall }}>>\rho_{u 0}$, equation (16) shows the void falling into complete emptiness at this $x_{e}$ coordinate.

$$
x_{e} \simeq \frac{\rho_{\text {wall }}}{\rho_{u 0}} x_{\text {wall }}
$$

This repartition of matter remains to be compared with experimental data [18]. But the novelty here is the existence of this stable equilibrium. It has no equivalent in the context of Newton's law. Of course, once the equilibrium obtained, the classical hydrodynamic equations still drive the behavior of matter for small and local perturbations.

Anyhow in a void, gravitational force is much stronger than that predicted by Newton's law. Supposing $\rho<<\rho_{u 0}$, equation (2) yields:

$$
C_{S M T} \cong \frac{\alpha_{0} \rho_{0}+\rho_{u 0}}{\rho_{u 0}}=\frac{2}{\Omega}=40
$$

The result is an evacuation of voids as soon as they are created. Collapse time in a void is now $t_{j}{ }^{\prime \prime}=20 \mathrm{~h}^{-1} \mathrm{Gyr}$.

As an intermediate conclusion, with SMT equations, the gravitational collapses are unaffected by expansion. The collapse time (equation (13)) is 2.4 times weaker than the predicted elapsed time since last decoupling (equation (10)). A stable equilibrium and an evacuation of voids are also predicted. This gives a possible explanation of the heterogeneities of large scale structure. 


\subsection{Galaxy dynamic}

This well-known mystery is, for example, evident in [19]. Simulations has been executed, based on [20] and [21]. Exactly the same initialization has been set, except that a greater initial mass and a smaller ray has been used in place of those used in [20].

Simulating immediately SMT model from [21] initial state results in a burst which greatly increases the disparity of stars velocities. To avoid this, SMT model is implemented progressively in the simulations, starting from Newton's model. The available data for calculating $C_{S M T}$ on each point of the galaxy, is the number of simulated stars $N b P$, which are located in the "SMT disk" of ray $r_{\max }$, centered on this point. And since the width of the galaxy is not easily available, the simulated volume matter density is not known. That's why the computed equations are the following.

$$
\begin{aligned}
& N b P_{\text {added }}=\frac{m_{0}}{m} \frac{h}{h_{0}} N b P_{0} \\
& N b P_{\mathrm{m}}=\max \left(N b P, \frac{7}{39} N b P_{\text {added }}\right) \\
& C_{S M T}=\frac{40 N b P_{\text {added }}}{39 N b P_{m}+N b P_{\text {added }}}
\end{aligned}
$$

$N b P_{\text {added }}$ is the constant which corresponds to 39 times $\rho_{u 0}$ in equation (2) and which has been progressively decreased during the simulations, starting from a very strong value. This progression is described below. $N b P_{m}$ corresponds to matter density in a galaxy, which is not exactly proportional to $N b P$, because $\rho_{I G M}$, IGM (intergalactic medium) matter density must be taken into account outside of the galaxy. It has been used $\rho_{I G M} / \rho_{u 0}=7$, and this specific value will be explained below in the study of virial theorem mysteries. $m$ is the mass of a simulated star. $m_{0}$ is the mass of a star which is used in the simulation of the Milky-Way, and which is therefore in accordance with MilkyWay's mass. $h$ is the width of the simulated galaxy's disk, supposed proportional to the size of the galaxy. $h_{0}$ is the width of the Milky-Way's disk. $N b P_{0}$ is the number of stars located in the SMT disk, at the sun's galactocentric distance of $8 \mathrm{kpc}$. Its value, 76000 , has been measured on the corresponding curve during the permanent regime of the MilkyWay simulation.

The program execution is divided into 2 phases. The first one is usual simulation of a virtual galaxy using Newton's law. This is done exactly like in [20], starting with the initialized galaxy described by the paragraph untitled "Initial conditions" in [21]. The end of this phase occurs after 50 galactic revolutions. At this time the $2^{\text {nd }}$ phase begins, in which Newton's law is replaced progressively by SMT. For ensuring this progressivity, the following equation is used, modifying equations (19).

$$
C_{S M T}=\frac{39 N b P_{\text {added }}+N b P_{\text {prog }}}{39 N b P_{m}+N b P_{\text {prog }}}
$$


At the beginning of this $2^{\text {nd }}$ phase, a very strong value (19 500000 ) is given to $N b P_{p r o g}$. Therefore at this time the simulation does not yield a great modification of the whole galaxy. The galaxy's shape is still very similar to the Newton's law permanent regime. Then, very slowly, $N b P_{\text {prog }}$ is decreased. Therefore, the shape of the simulated galaxy slowly changes. This decrease stops as soon as $N b P_{\text {prog }}=N b P_{\text {added }}$ is reached, after 750 revolutions. Therefore at this time the full equations (19) are finally computed. Figure 2 shows the results. For comparison, figure 1 shows exactly the same initial galaxy, after the same number of revolutions, but always simulating Newton's law. The galactic center shows the apparition of a ring [22]. This is discussed below. Newtonian logarithmic matter density profile is curved positively, from 0 to $50 \mathrm{kpc}$ galactocentric distances. But SMT one is a straight line between 20 and $70 \mathrm{kpc}$. This is more compatible with experimental data. But of course, below $20 \mathrm{kpc}$, the curve is no longer a straight line due to the existence of the ring. Radial and tangential speed dispersions are 2 or 3 times worse than the one obtained with Newton's law.

No stable spiral arms are noticed, like in [20]. Like in [20], they can appear only from time to time and are not stable structures. But the kind of apparition of arms shown by figure 4 seems to be provoked by the overall increase of $C_{S M T}$ though the whole galaxy. A Keplerlike speed profile is of course shown under Newton's law (figure 1). Those speed profiles are completely ruled out by experimental data, as commonly accepted. But SMT speed profiles are much closer to flat curves. Of course this comes from the "smoothing" behavior of SMT model, on velocities: $C_{S M T}$ increases with distance from the galactic center, due to matter density decrease. The galaxy speed profile of figure 2 has a shape which can be easily compared to the Milky-Way speed profile shape, for example. The speed profiles yielded by SMT are always far closer to experimental ones than those yielded by Newton's law. This was true for each executed simulation, which were done using various values of SMT parameters (an $N b P_{\text {added }}$ different value, and also other constants than $7 / 39,40$, 39 ). The values of those parameters in equations (19) are predicted by SMT but are not the most appropriate in order to yield the best profiles when comparing to experimental data. Nevertheless in this document, the simulations are always computing equations (19), except when expressly mentioned. Assuming different values for the parameters than those in equations (19), an almost perfectly flat speed profile can be obtained, for giant galaxies. This is also very much compatible with experimental data. Indeed, such a flat profile is observed in the case of UGC 2885 [23], NGC 801, or NGC 2403, for instance. Assuming different values for the parameters a typical "increasing bell shape" profile can also be obtained, for smaller galaxies. This is obtained in particular when the ring is weak, or absent. Finally, a little "wave" is often noticed at the beginning of the speed profiles, which is often present in experimental speed profiles. For example, on figure 2 this wave is located around $10 \mathrm{kpc}$ from the galactic center.

Galaxy stability is increased in lower density environments for medium- or large-sized galaxies. Indeed, it has been supposed when calculating equations (19), that IGM matter density is equal to $\rho_{I G M}=7 \rho_{u 0}$. But supposing $\rho_{I G M}=0$ implies a modification of equations (19), such as $N b P_{\mathrm{m}}=\max \left(N b P,(7 / 39) N b P_{\text {added }}\right)$ is replaced by $N b P_{\mathrm{m}}=N b P$. This increases the maximum $C_{S M T}$ possible value from 5 to 40 . This 
multiplication of gravitational forces by a factor of 8 , valid only when located out of the galaxy (or at the edge of it) will of course increase its stability. This has been confirmed by simulations (under the $h=h_{0}$ hypothesis) and might be in accordance with experimental data [24]. In this $\rho_{I G M}=0$ case, after its slow dissolution, a giant galaxy yields a very faint large galaxy, which can be easily compared with a LSB galaxy. But this does not occur under the $\rho_{I G M}=7 \rho_{u 0}$ supposition, in which case this galaxy is only dissolving faster, without any remaining structure.

The computational flexibility is low and the liberty degrees are in the galaxy characteristics input. The first characteristic is the simulated matter density with respect to $\rho_{0}$, in other words $N b P$ with respect to $N b P_{0}$. This depends on parameters such as the width and volume matter density in a galaxy, with respect to its size. The second characteristic is the exact knowledge of gas and star distribution in the galaxies. This gas distribution was not taken into account in those simulations. Hopefully, in spiral galaxies the gas density is only a few percent of that of stars, and in elliptical galaxies it is even lower. Therefore this error might have no strong effects. But this might not be true for simulating a standalone dwarf galaxy. Anyhow this gas behavior simulation would require a specific kind of computation. The issue of this chapter is the most difficult and delicate. It would need a huge amount of work. But even without such a workload, here the SMT results speak for themselves.

\subsection{Dwarf galaxies}

The simulations show an occasional generation of dwarf galaxies, orbiting around the main galaxy. But this occurs only when simulating under SMT model. When simulating Newton's law, no dwarf galaxies were noticed. Dwarf galaxies are almost systematically generated during the burst which occurs at the start of the simulation, when SMT model is immediately fully calculated, without beginning with a first phase in which Newton's law is active. Dwarf galaxies may also be generated with a simulation using a progressive installation of SMT model calculation. In this case, they can appear just at the beginning of the second phase of the simulation, which is executing progressively SMT model.

A faint galaxy, because it is faint, is probably located in a low or very low matter density environment. Therefore, $C_{S M T}$ is strong and the perceived gravitational attraction is high (may be up to 40 times greater than Newton's law attraction). As a result, the existence of this faint dwarf is more understandable under SMT than under Newton's law, as well as its important velocity in its revolution around a main galaxy. In front of this, faint and ultrafaint galaxies existence could be an issue under MOND theory [25]. The same mechanism might explain the mystery of "the lower the surface brightness of a system, the larger its mass discrepancy [26]". Indeed, low surface brightness galaxies are mainly isolated field galaxies. As such, they might also be located in a low matter density environment.

Those dwarf galaxies 2D generations are in accordance with experimental data. Indeed, studies of M31 [27], of the Milky-Way [28], and even of globular clusters and streams around the Milky-Way [28] has shown a systematic preferred location of dwarf galaxies along a common disk. Of course, it would be better to simulate in $3 \mathrm{D}$, but the existence of $2 \mathrm{D}$ generations itself might be a result since no such dwarf galaxies were generated by the same simulations under Newton's law. After a while they often dissolved progressively by themselves. When the main galaxy contains a ring, those dwarf orbiting galaxies are generated only outside of this ring, therefore at more than $15 \mathrm{kpc}$ from the galactic center. 

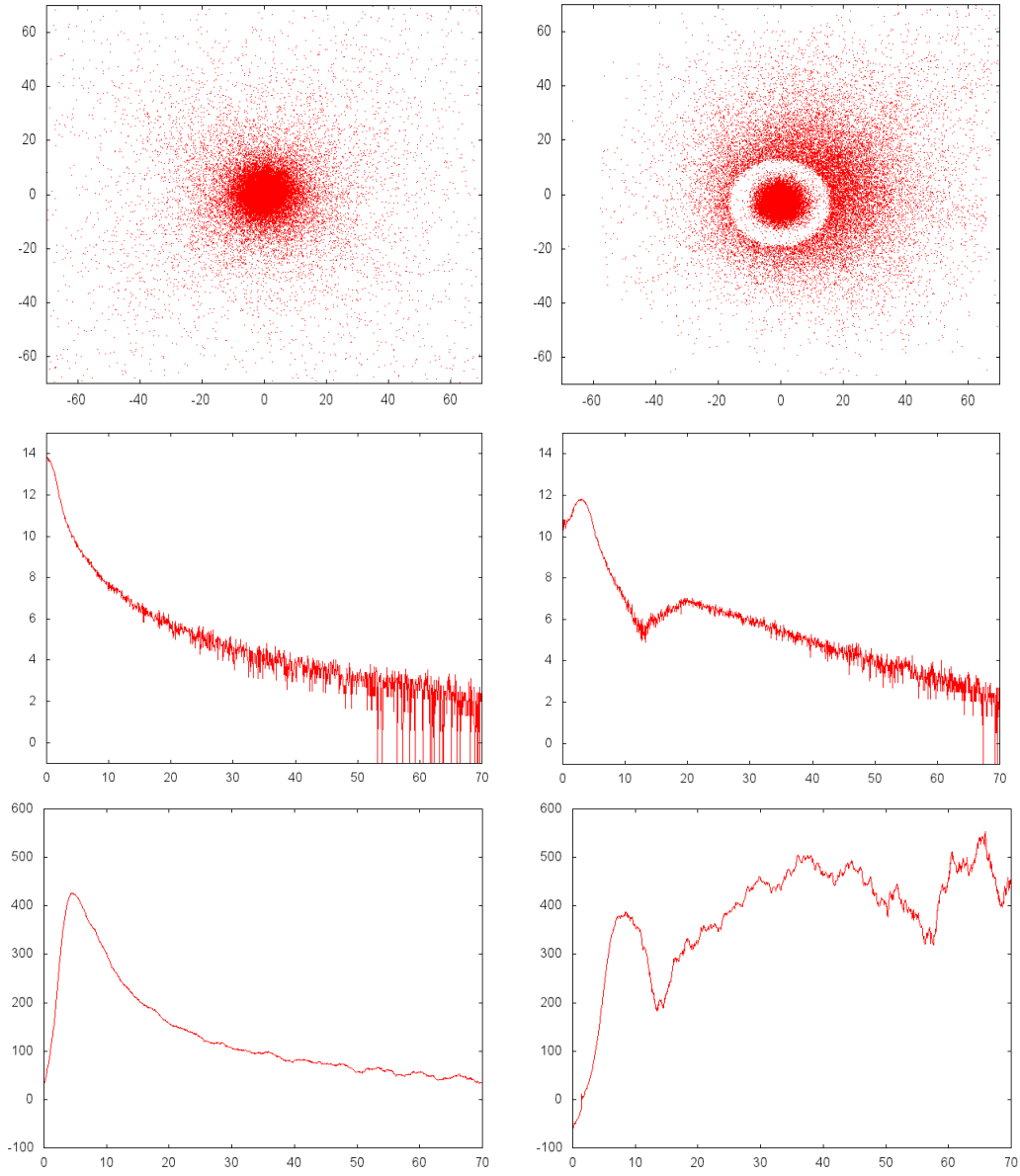

Figure 1: On the left are shown the results of a simulation executed under Newton's law. The simulated galaxy is the same as in [20] after 750 revolutions, except that the galaxy mass is equal to $1.810^{12}$ sun masses, the initial galaxy ray is equal to $1.4 \mathrm{kpc} .10^{5}$ stars has been simulated in a $256 \times 256$ grid calculation over a $67 \times 67 \mathrm{kpc}^{2}$ square. On top the $2 \mathrm{D}$ localization of the stars is drawn. In the middle $8+\log (\rho)$ is drawn, where $\rho$ is the mean value over $13 \mathrm{kpc}$ of galactic surface matter density. $\rho$ unit is $\mathrm{kg} / \mathrm{m}^{2}$. On the bottom the tangential speed of the stars in $\mathrm{km} / \mathrm{s}$ is drawn. For those three drawings, horizontal values (and vertical values for the top drawing) are galactocentric distances, with unit in $\mathrm{kpc}$,

On the right are the results of another simulation, which is the same as the one represented on the left, except that SMT model has been progressively introduced in place of Newton's law, Finally those results are obtained, after 750 revolutions, with an exact SMT simulation (equations (19)). The same axis and units are used as on the left. 
It was noticed also that they are often encountering the galactic center along their trajectory. But when this center contains a ring, which is often the case, they cannot dissolve themselves into it. They are systematically bouncing on the ring edge, forbidding any merge. This might result in a better stability of dwarf galaxies, with SMT, than with Newton's law.

By other means, the location of the Milky-Way on the plane containing the dwarf orbiting galaxies around M31 [27] is not explained by the model and would need a 3D simulation.

\subsection{Ring galaxies}

During the simulations a surprising result was found. Ring galaxies are oftenly generated by the SMT dynamic itself, without the help of any particular external event. They appear to be self-generated and stable structures. There is no longer the need to imagine any collision scenario between galaxies in order to explain their existence. And this is in complete accordance with experimental data. For instance, in a region called "the general field galaxy population lying behind the Tucana dwarf galaxy" [29], an "unexpectedly large number of ring galaxies" is found. This number is inconsistent with the hypothesis of ring galaxies generated only by collision. SMT might predict this abundance directly, without supposing any "steeply increasing galaxy interaction rate with red-shift" [30]. Also, it could be difficult to understand why those ring galaxies are so young [31]. But this mean age of $1 \mathrm{Gyr}$ might be understood by the simulations. Indeed, several dislocations of rings were noticed during the simulations. Those dislocations were always very quick and resulted in the same galaxy but without any more ring. This could indicate that nevertheless this stability is fragile, and therefore could explain this relatively young age. Even our MilkyWay galaxy has been recently found to host a "ring of stars" [22].

When simulating a galaxy with the same characteristics (mass, star velocities, diameter) as the Milky-Way, this ring is found. It has a radius of $18 \mathrm{kpc}$, which is exactly the experimentally observed ring radius. Also, the simulations has shown multi-ring stable structures. When simulating equations (19) they are noticed for big galaxies (having a ray greater than $50 \mathrm{kpc}$ ). This is confirmed by experimental data. For example NGC 2859 double ring is obvious. The simulated ring shows strong matter density values also beyond the ring itself. This is not consistent with the visible rings of observational data. But as explained in [32], there is probably a low luminosity gaseous disk in ring galaxies.

The simulations seem to show that SMT prediction is the following. The width of the empty ring could not be greater than $r_{\max }$. Moreover, it should be often below or just below this value. The simulations, for example the one of figure 3, shows a ring diameter of $34 \mathrm{kpc}$, composed of a nucleus diameter of $10 \mathrm{kpc}$, and an empty ring width of $12 \mathrm{kpc}$ (just below $r_{\max }$ value). Those dimensions are similar to the Cartwheel galaxy dimensions, for example. 


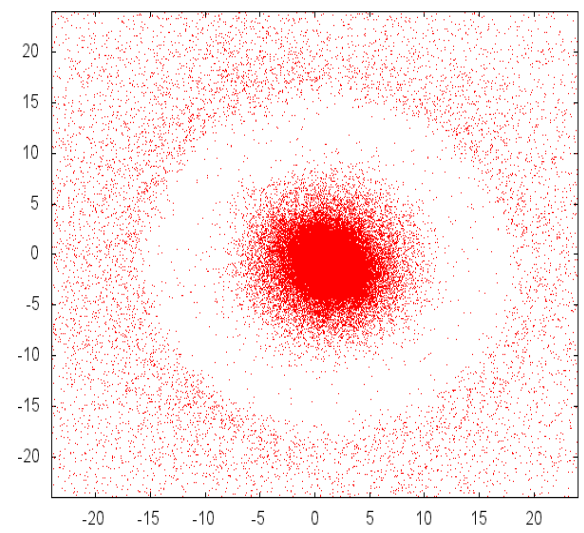

Figure 3: A simulated ring galaxy

The galaxy simulations shows that the ring's ray of the simulated galaxies, when they contain a ring, is always just below $r_{\max }$. Therefore an attempt could be to retrieve on existing galaxies the statistical distribution of such a ray.

The simulation has shown that the particular truncated shape of the observed bars in the galaxies, along with the particular enrolled shape of arms around it, can result from the existence of a ring in those galaxies. For instance, on figure 4 the length of the bar is roughly equal to $35 \mathrm{kpc}$. This bar is the nucleus of an ancient ring galaxy. This nucleus started to deform itself and to rotate quickly. As a result the generated arms quickly enrolled around it. The result is then shown on the figure.

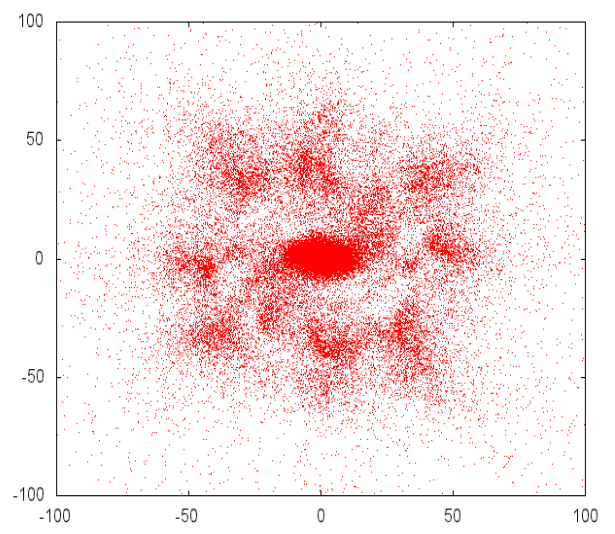

Figure 4: This galaxy shape appears when executing equations (20) with

$N b P_{\text {prog }}=705000, N b P_{\mathrm{m}}=N b P$, and equations (20) with $h=h_{0}$. At this time $N b P_{\text {prog }}$ is decreasing at a pace of 15600 per galactic revolution. The central bar is rotating quickly, enrolling 2 arms around it. The simulated galaxy has a mass equal to $1.510^{14}$ sun masses, and an initial ray equal to $1.9 \mathrm{kpc}$,

The similarity with observations is noticeable. And this was not obtained when simulating Newton's law with the same program. With Newton's law, the bar and its arms always showed a "slow S", as in [19]. This particular observed shape described above was never noticeable neither for the bar nor for the arms, under Newton's law.

Finally another weird prediction of the simulations is that the rotational speed of the stars located in the nucleus is sometimes opposite to the speed of the stars located outside of the ring. This prediction is validated by NGC 7217 speed profile.

Of course, this work is still in progress. A 3D simulation is needed, in order to know if the exact prediction is a ring, or a radial hole. And this simulation must be done using real 
galaxy data, and then compared with observations. Finally, comparing this with the corresponding MOND and $\Lambda \mathrm{CDM}$ model predictions would be very interesting.

\subsection{Galaxy mass and scale}

The observational fact that galaxy masses are proportional to their size [33], is not predicted immediately by the model. It would require a deeper understanding of galaxy dynamics in the context of SMT.

\subsection{Virial theorem}

Let's try to quantify what predicts the model in the cases studied by [34], [35] (gravitational forces greater than expected in Virgo or Coma clusters, among others).

It will be assumed $\rho_{I G M} / \rho_{u 0}=7$, which is roughly the lowest of the commonly accepted bounds $(6,200)$ [36] for this ratio. Equation (2) yields the following value for the SMT increase of gravitational forces in IGM:

$$
C_{S M T}=\frac{\alpha_{0} \rho_{0}+\rho_{u 0}}{\rho_{I G M}+\rho_{u 0}}=5
$$

This equation yields the measured value of 5. Therefore with SMT it might be possible to explain the virial theorem classical mystery. Also, other gravitational anomalies like the bending of light, or the "Great Attractor" [37] anomalies might be explained, exactly the same way.

\subsection{Bullet cluster}

This is "1E 0657-56" galaxy cluster [38], [39], [40]. Possible explanations of its strange dynamic have been given for example in [41] and [42]. The main cluster's length is around $700 \mathrm{kpc}$. With $r_{\max }=15 \mathrm{kpc}$, this makes a relative value of $2 \%$. That's why the probability of intersection of a galaxy with the SMT sphere has been approximated to 0 . But of course this must be confirmed based on a detailed information about the bullet cluster. Hence, only the gas cloud has been taken into account for evaluating $\rho$ in equation (2). A simulation of SMT prediction has been executed. It has been supposed a plane distribution of matter (on the plane which is perpendicular to the line of sight). Therefore only a $2 \mathrm{D}$ simulation has been executed. Of course a $3 \mathrm{D}$ simulation would be better. But this simplification allows the SMT dynamic to be easily perceived in such a case. A Gaussian distribution of matter has been used for each of the two clusters, and for the gas distribution. The fitted standard deviations of the gas cloud and the clusters distributions has been set respectively to $560 \mathrm{kpc}$ and $130 \mathrm{kpc}$. Their fitted relative amplitude has been set respectively to 1 and 0.3 . The distance between the centers of the two clusters has been set to $750 \mathrm{kpc}$. For simulating equation (2), the simulated matter density, $\rho$, has been multiplied by a fitted constant. It has been supposed also $\rho_{u}=\rho_{u 0}$. Then, the calculation of the algorithm was the following. Based on those mass distributions, the acceleration potential is calculated. In the case of figure 5, it is Newton's potential. In case of figure 6, it is SMT potential given by equation (2). Then, mass distributions are 
calculated (back) again, based on those potentials. But now for figures 5 and 6 , those mass densities are calculated supposing that the potential $\phi$ is a Newtonian potential. A precise FFT is not available, therefore, the usual Poisson formulation of Newton's law is used: $\Delta \phi=4 \pi G \rho_{f}$, where $\rho_{f}$ is the final mass density shown on the figures. The numerical calculation of this $\Delta \phi$ Laplacian uses the following matrix.

$$
A=\left(\begin{array}{ccc}
0.4 & 0.2 & 0.4 \\
0.2 & -2.4 & 0.2 \\
0.4 & 0.2 & 0.4
\end{array}\right)
$$

This is calculated with a 64 bits floating point format on a $256 \times 256$ grid covering the bullet cluster. It yields visible errors in the final result. But on figure 5 this calculated mass distribution is qualitatively the same as the initial one, and the interesting result is obtained when comparing figure 5 with figure 6 . Figure 6 reveals the following SMT mechanism. Mainly, $C_{S M T}$ is inversely proportional to local gas matter density. In the clusters, since there is almost no more gas among the galaxies, this factor is strong. Conversely, between the two clusters, in the gas cloud, it is lower. This explains qualitatively the bullet cluster weak lensing mass distribution. More precisely, based on equation (2), SMT predicts that $\Phi_{g}(M)$, the SMT acceleration potential generated by the gas cloud only, and applied on a given $M$ point, is proportional to the following expression.

$$
\Phi_{g}(M) \propto \frac{\frac{G}{d(M, N)} * \rho_{g}(N)}{D_{M}(N) * \rho_{g}(N)}
$$

$\rho_{g}(N)$ is the gas density located on a given $N$ point. $d(M, N)$ is the distance between $M$ and $N . D_{M}(N)$ is equal to 1 if $d(M, N)<r_{\max }$, else it is equal to 0 .

When comparing the bottom of figure 6 with figure 1a of [38], the 2D whole profiles are qualitatively the same and many similarities are noticed. On those two figures the gas cloud seems to generate no Newton-like gravitational attraction. This is predicted by equation (23)23, because $G / d(M, N)$ and $D_{M}(N)$, as functions of $N$, are symmetrically centered, and thin. This is also because $\rho_{g}(N)$ varies on a $700 \mathrm{kpc}$ scale, and $r_{\max }<<700 \mathrm{kpc}$. The result is that equation (23)23 shows nearly no variation of $\Phi_{g}(M)$. Due to this specific quotient of convolutions yielding $\Phi_{g}(M)$, it might even be predicted other similarities with the experimental figure. Noticeably, on figure $1 \mathrm{~b}$ of [38], the mass contours on the left are following the end of the gas cloud limits. Also, the mass contours tend to surround closely the two bowls of gas (in black). This could be predicted because $G / d(M, N)$ and $D_{M}(N)$, although symmetric and thin, are nevertheless different. Noticeably, $D_{M}(N)$ has finite support whereas $G / d(M, N)$ 
has not. Moreover, on the left of figure 6 , the mass contour predicted by SMT draws an " $\mathcal{~}$ " shape. This shape is quite similar on figure $1 \mathrm{~b}$ of [38]. Finally, a small valley can be seen, between the two clusters (top center of figure 1a of [38]). This valley is also present in figure 6, as well as another one just below. Different simulations shows that those two symmetrical valleys are a systematic result of SMT when varying the parameters of the modelled mass distribution.

Of course, this simulation would better be executed in 3D. It would better use real experimental data, such as real matter densities, as well as the exact galaxy locations and dimensions. But the overall result is that SMT suggests a possible explanation of bullet cluster weak lensing mass reconstruction.

Concerning the strange relative velocity of the clusters [43], [44], equation (18) shows that gravitational acceleration can increase by a $C_{S M T}$ factor of up to 40 . This value, valid inside a void, is much greater than the corresponding values of $\Lambda$ CDM and MOND models [44], which are in the range $(2-6)$. For example, a cluster located in a void can be strongly accelerated by the neighboring walls, during the predicted evacuation of the void. Therefore, this cluster will receive an acceleration much greater than the one coming only from any other cluster. In fact, this acceleration is generated by the attracting walls, not only by the other cluster. And, also, $C_{S M T} \cong 40$ is valid in this case. The result is that the relative velocity between the two clusters might be much greater than the one generated only by their own masses, and much greater than Newton's law prediction. Therefore, the SMT predicted velocity might be in accordance with the observed value of $4700 \mathrm{~km} / \mathrm{s}$ [44]. And for the same reasons, SMT might predict that such a cluster velocity would not be a rare event [43]. But of course this has to be confirmed by precise calculations or simulations.
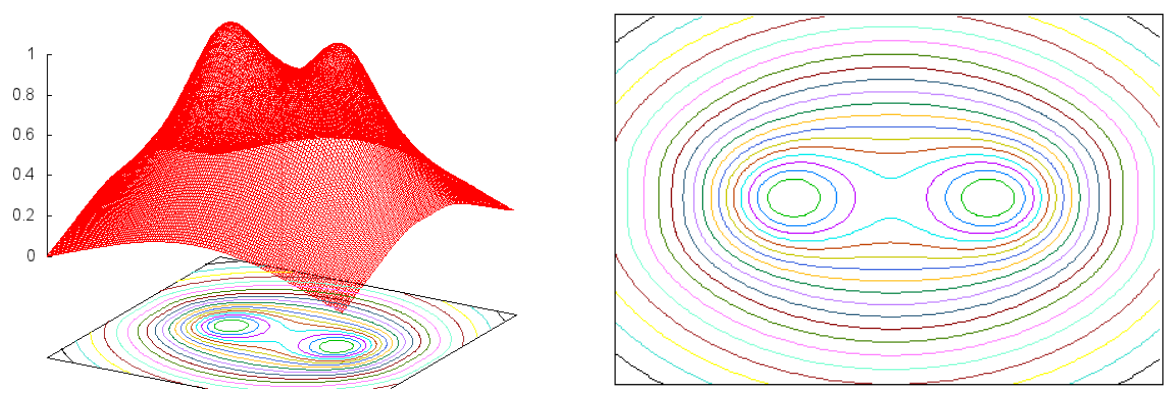

Figure 5: On the left are drawn in 2D the contours of the 3D surface which is shown on the right. It represents a modelled mass distribution of the bullet cluster. Three gaussian distributions have been used for modelling the two galaxy clusters and the gas cloud. The result shown is the calculated Laplacian of the potential. This potential has been calculated using Newton's law, based on this mass distribution. This figure is here for comparison with figure 6 . This final result is slightly different from initial mass distribution due to program execution precision errors. Vertical units are relative matter density. 

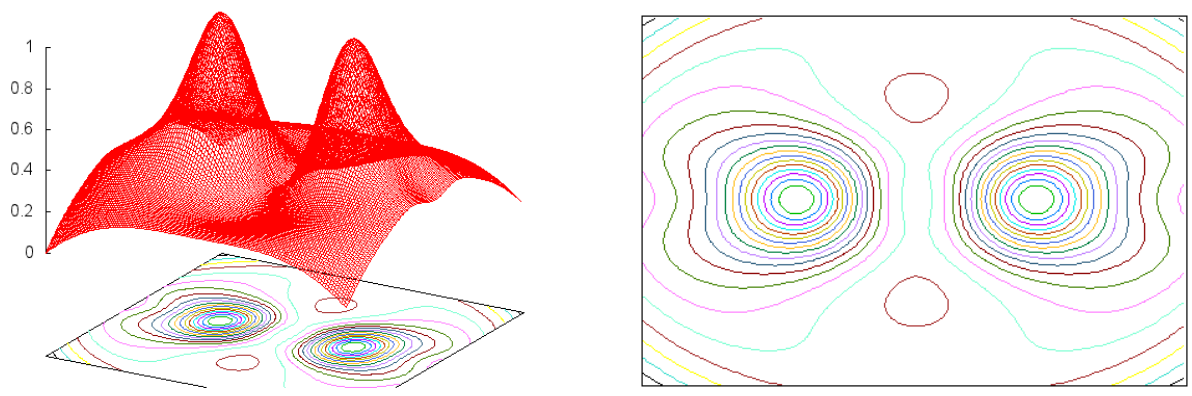

Figure 6: On the left are drawn the $2 \mathrm{D}$ contours of the $3 \mathrm{D}$ surface which is shown on the right. The same initial mass distribution has been used as for drawing figure 5. The shown result is the calculated Laplacian of acceleration potential. This potential has been calculated using SMT, based on this initial mass distribution. This figure must be compared with figure 5 and uses the same units.

\subsection{Tully-Ficher law}

Trying to explain Tully-Ficher law [45] in the context of SMT leads to the conclusion that $\alpha$ in a galaxy depends on the galaxy characteristics. This is of course not satisfying. But a slight modification of the model is possible, yielding the exact Tully-Ficher relation. This work is in progress.

\subsection{Miscellaneous}

The tremendous amount of gravitational mysteries [46], [47], are not easy to address in one article. For example, simulation of the galaxy interactions and mergers, such as the Antennae [25], would be interesting. But some issues listed in [47] are already qualitatively answered in the context of SMT. Among others, each of the following issues might be explained simply by some of the basic SMT mechanisms which have been described in this document.

- Features in the baryonic distribution imply features in the rotation curve.

- The bulk flow challenge.

- The high-z clusters challenge.

- The Local Void challenge.

- a0/G as a critical mean surface density for stability.

- $\mathrm{a} 0$ as a transition acceleration.

The issue titled "Features in the baryonic distribution imply features in the rotation curve" seems easy to solve in the context of SMT since it's a modified gravity theory, not a Dark Matter theory.

The "bulk flow challenge" might be qualitatively answered exactly the same way as above, for the measured relative velocity of the sub-clusters of the bullet cluster.

The "high-z clusters challenge" is answered simply by equation (10): the time since last scattering is $68 / 9.35 \cong 7$ times greater than $\Lambda C D M$ one. Existence of big structures at high red-shift is possible with SMT.

The "Local Void challenge" issue, which asks why the local void, located around the Milky-Way, has so few galaxies, has a simple direct explanation in the context of SMT. Indeed, as stated above, in a void, the applied gravitational force is up to 40 times greater 
than Newton's one. Therefore, the remaining galaxies are strongly attracted to each other, resulting in a quicker creation of giant galaxies. Another SMT behavior is that those giant galaxies are more stable in a low matter density environment. This mechanism has been explained above. Also, the main proportion of matter in a giant galaxy is not located on its edge. Therefore, $C_{S M T}$ can stay close to 1 in an inner and major part of the galaxy. As a consequence, a giant galaxy might not follow the evacuation of a void, which drives only sparsely distributed and isolated objects. The final result is a low number of galaxies, and a higher proportion of giant galaxies predicted by SMT in low matter density environments. And exactly this is shown by experimental data [24].

The issue untitled « a0/G as a critical mean surface density for stability" has been partially explored when simulating the galaxies. Indeed, figures 1 and 2 shows that matter density of the same virtual galaxy in a Newton's context has its maximum more than $10^{2}$ times greater than the corresponding one in SMT. This is due to the strong SMT decrease of gravitational forces at locations where matter density is strong. This creates a cutting off behavior of SMT for strong matter density values. And it might be guessed that this implies a decrease of a galaxy mean surface density between the two models. This might give a first answer to this issue.

The issue untitled "a0 as a transition acceleration" is well resumed by figure 48 of [47]. It shows that the mean acceleration inside galaxies and clusters stays roughly constant, independent of the scale. This might be a consequence of the existence of the cutting-off value, suggested above.

By the way, GR singularities and closed time-like curves can be addressed by SMT for the same reason. Indeed, under SMT context, very high matter densities are much harder to occur than under GR, because of the cutting-off value suggested above.

SMT predicts also a varying, or, different orbital period for binary stars and exoplanets (different than Newton's law prediction). This must be calculated, using a measurement of the surrounding matter density in each case. But this might give an explanation of the issue of the strange variation of orbital period of black hole X-ray binaries [48].

\section{Violation of momentum conservation at large scale}

In the context of SMT, the principle of momentum conservation is violated at large scale. Indeed, equation (2) shows that two mutually attracted objects might not exert on one another exactly opposing gravitational forces. But this prediction concerns only objects attracted to one another that are more than $15 \mathrm{kpc}$ apart.

Comparing this prediction with observational data, for example in the case of clusters of galaxies, is extremely important. But this violation appears only when matter density is varying consistently. Hence it would need precise calculations based on detailed information on gas and stars distributions.

\section{Testing the model}

\subsection{Intragalactic}

This test consists in continuing the simulation of a galaxy under the SMT model. A specific focus on flat profiles of giant galaxies might be done. 


\subsection{Extragalactic}

One test could be to measure matter density and a possible increase of gravity, outside of any galaxy. Indeed, the following equation is the rewriting of equation (2) for extragalactic spaces.

$$
\frac{G^{\prime}}{G}=2 \frac{\rho_{c}}{\rho+\rho_{u}}
$$

Let's remind the notations.

$G$ is the gravitational constant.

$G^{\prime}$ is the equivalent value of $G$ as predicted by the model. Therefore $G^{\prime} / G$ is the increase of the gravitational force, but at a given $\mathrm{M}$ space-time location, as predicted by the model. M must be located outside of any galaxy. It is the increase of $G$ which must be used for calculating any exerted force in M. It is not a generated one, because the generated ones are simply using $G$, that is, unmodified Newton's law.

$\rho$ is the matter density calculated in $10 \mathrm{~h}^{-1} \mathrm{kpc}$ ray sphere centered in $\mathrm{M}$.

$\rho_{u}$ is the mean matter density of the Universe at the time of the M event.

$\rho_{c}$ is Universe critical matter density.

This equation shows a big difference with $\Lambda \mathrm{CDM}$, in the case of voids: $G^{\prime} / G \simeq 40$ if $\rho=0$. More generally, the model predicts a difference with Newton's law in areas in which $\rho=\rho_{I G M}$ varies consistently.

\subsection{Large scale structure}

This is the test of the very SMT specific stable equilibrium. It is predicted by SMT over large scale structure. Either equation (16) or equation (17) might be used.

The test might consist in measuring the observed matter density distributions which are used in those equations, and then to check if those equations are retrieved.

\section{Conclusion}

SMT is composed of equation (2) and of 2 parameters, $r_{\max }$ and $\alpha$. Each gravitational mystery, or any gravitation behavior acting below $r_{\max } \cong 15 \mathrm{kpc}$ from the sun does not have to be checked by the model. Indeed, for those cases the model behaves exactly like Newton's law. The overall behaviour of this model shows gas and dust playing a major role in the strength of the perceived gravitational force. First of all they generate Newton-like gravitational attraction like any other energy. This is done at the location where gravitational force is created (generated). But also, the presence or absence of gas or dust at the location where gravitational force is applied (exerted), respectively decreases or increases its strength.

This is the main novelty of this model. It might explain, in a simple manner and quite directly, the virial theorem mystery, and the bullet cluster issue. It might explain also the strong relative velocity of the sub-clusters of the bullet cluster, and similar issues. On 
cosmological scale SMT predicts that gravitational forces are inversely proportional to Universe density. This leads to a de Sitter Universe with $K=0$ and $q=-1$, which is compatible with measured values. The value of critical Universe density, fine tuning, and the issue of particle's horizon are in accordance with this de Sitter behaviour.

Big bang singularity is avoided by SMT. Complete emptiness, or infinite matter densities lead to an infinite equivalent $G$ for the former case, and to a null equivalent $G$ for the latter case. This appears to be compatible with Mach's principle. But primordial nucleosynthesis is not predicted by SMT. This shows that, however, a more sophisticated model could be required in order to model the microscopic scale.

SMT might explain heterogeneities of large scale structure and galaxy formation. This is because the predicted time since last scattering is 7 times greater than with $\Lambda$ CDM. But this is mostly because with SMT, the effect of matter density is independent of the scale factor. An explanation of the voids emptiness might also be given by the prediction of a specific equilibrium.

Galaxy speed profiles are complex to work on because they need an important and detailed experimental data. They also require a huge amount of work. But the predicted speed profiles seems to be closer to experimental ones than Newton's law prediction. Varying SMT parameters, each kind of experimental speed profile might be actually retrieved by a simulation of a virtual galaxy. Even very flat curves of giant galaxies seems to be retrieved. The structure and dynamic of the galaxies seem compatible with experimental data. An unexpected prediction is also found by the model's simulations: ring galaxies are easily created by SMT specific dynamic, in a stable state. Their structure, frequency and age might be in accordance with experimental data. The "Local Void" challenge might be solved qualitatively as well as the "lower the surface brightness of a system, the larger its mass discrepancy".

SMT predicts exactly the same behavior as GR, but with a possibly different and constant value of $G$, in each cases where matter density stays constant (evaluated in the $15 \mathrm{kpc}$ ray SMT sphere). Conversely in each case in which this matter density consistently varies, SMT predicts a variation of $G$. In those cases the variation of a dilatation factor is also predicted, acting on the space components of the stress-energy tensor. This remains to be thoroughly calculated and compared with experimental data. The only required test in solar system is testing the variation of $G$ over more than 50000 years. Outside the solar system, testing any modified $G$ must be done only $15 \mathrm{kpc}$ beyond solar system.

The Tully-Ficher law and the linear variation of galaxy mass with size are not explained.

A scientific comparison of the model's predictions with experimental data must be done. And this has not even been started here. But nevertheless this work could suggest that the new kind of dynamic yielded by SMT might help to understand some of the today's gravitational mysteries.

\section{Acknowledgments}

I acknowledge discussions with Craig Sarazin.

\section{References}

[1] C.H. Brans, arXiv:gr-qc/0506063v1 (2005).

[2] L. Susskind, Foundations of Physics (2011). doi:10.1007/s10701-011-9620-x 
[3] R.J. Gaitskell, Ann. Rev, Nuc. Part. Sci. 54, 315-59 (2004).

[4] R.H. Sanders, S.S. MacGaugh, Ann. Rev. Astron. Astrophys. 40, 263-317 (2002).

[5] J.D. Bekenstein, Phys. Rev. D70, 083509 (2004).

[6] J.D. Bekenstein, R. H. Sanders, Astrophys. J. 429, 480-90 (1994).

[7] C. Roychoudhuri, Journal of Modern Physics, Vol. 3 No. 10, pp. 1357-1368 (2012). doi: $10.4236 /$ jmp.2012.310173.

[8] J. Leibovitz, Journal of Modern Physics, Vol. 2 No. 12, pp. 1470-1479 (2011). doi: 10.4236/jmp.2011.212181.

[9] M. Salgado, D. Martinez-del Rio, Journal of Physics: Conference Series 91 (2004). doi:10.1088/1742-6596/91/1/012004.

[10] B. Canzian, R. J. Allen, R. P. J. Tilanus, Astrophys. J., Part 1, vol 406 (1), no 2, p 457 469 (1993).

[11] B. Bouwens et al., arXiv:1211.3105 [astro-ph.CO] (2013).

[12] G. B. Brammer et al., The Astrophysical Journal Letters, 765:L2 (2013). doi:10.1088/2041-8205/765/1/L2.

[13] J.R. Gott, J.E. Gunn, D.N. Schramm, and B.M. Tinsley, Astrophys. J. 194, 543-553 (1974).

[14] P. de Bernardis et al., Am. Inst. Phys. Conf. Proc. 555, 85-94 (2000).

[15] A. Riess et al., Astron. J. 116, 1009-1038 (2009).

[16] P. de Bernardis et al., Nature, Vol 404 (2000).

[17] S. A. Shectman, S. D. Landy, A. Oemler, D. L. Tucker, H. Lin, R. P. Kirshner, P. L. Schechter, Astrophys. J. 470, 172-88 (1996).

[18] R.B. Tully, In IAU Symposium, edited by J. Davies \& M. Disney, vol. 244 of IAU Symposium, 146-151 (2008b).

[19] T.S. Van Albada, and R. Sancisi, Phil. Trans. R. Sac. Land, 320, 447-464 (1986).

[20] F. Hohl, W. Hockney, Journal of Computational Physics, 4, 306-324 (1969).

[21] F. Hohl, The Astrophysical Journal, 168:343-359 (1971).

[22] B. Yanny et al., Astrophys. J., 588:824-841 (2003).

[23] V. C. Rubin et al., The Astrophys. J., 238:471-487 (1980).

[24] P.J.E. Peebles and A. Nusser, Nature, 465, 565-569 (2010). doi:10.1038/nature09101

[25] O. Tiret, F. Combes, Formation and Evolution of Galaxy Disks ASP Conference Series, Vol. 396, Proceedings of the conference held 1-5 October 2007. Edited: Astronomical Society of the Pacific, p.259 (2008).

[26] W.J.G. de Blok and S.S. McGaugh, ApJ 508132 (1998). doi:10.1086/306390.

[27] R. A. Ibata, Nature, 493, 62-65 (2013). doi:10.1038/nature11717.

[28] P. Kroupa et al., Astron. Astrophys., 523, A32 (2010).

[29] M. Pawlowski, J. Pflamm-Altenburg, P. Kroupa, Mon. Not. R. Astron. Soc., 000, 121 (2012).

[30] R. J. Lavery et al., The Astrophysical Journal, 467 :L1-L4 (1996).

[31] B. C. Whitmore, R. A. Lucas, D. B. McElroy, The Astronomical Journal, Vol 100, n 5 (1990).

[32] B. C. Whitmore, The Astrophysical Journal, 314:439-456 (1987).

[33] J. P. Ostriker, P. J. E. Peebles, and A. Yahil, Astrophys. J. 193, L1-L4 (1974).

[34] F. Zwicky, Act. Helv. Phys. 6, 110-127 (1933).

[35] F. Zwicky, Astrophys, J. 86, 217-246 (1937).

[36] T. Fang et al., arXiv:1001.3692 [astro-ph.CO] (2010).

[37] R.C.Kraan-Korteweg, arXiv:astro-ph/0006199 (2000).

[38] M. Markevitch, A. H. Gonzalez, D. Clowe, A. Vikhlinin, W. Forman, C. Jones, S. Murray, W. Tucker, ApJ. 606, 819 (2004).

[39] D. Clowe, A. H. Gonzalez, M. Markevitch, arXiv:astro-ph/0312273v1 (2003).

[40] D. Clowe, M. Bradac, A. H. Gonzalez, M. Markevitch, S. W. Randall, C. Jones, and 
D. Zaritsky, ApJ. 648, L109 (2006).

[41] J. R. Brownstein and J. W. Moffat, Mon. Not. R. Astron. Soc., 382, 29-47 (2007). doi:10.1111/j.1365-2966.2007.12275.x.

[42] M. Bradac et al., ApJ. 652, 937 (2006).

[43] E. Hayashi, S. White, Mon. Not. R. Astron. Soc., 370, L38 (2006). doi:10.1111/j.1745-3933.2006.00184.x

[44] G. W. Angus and S. S. McGaugh, Mon. Not. R. Astron. Soc. 383, 417-423 (2008). doi:10.1111/j.1365-2966.2007.12403.x.

[45] Tully, R.B. and Fisher, J.R., Astron. Astrophys., 54, 661-673 (1977).

[46] B. Famaey, S. McGaugh, arXiv:1112.3960 [astro-ph.CO] (2012). doi: 10.12942/lrr2012-10.

[47] B. Famaey, S. McGaugh, J. Phys.: Conf. Ser. 437012001 (2013). doi:10.1088/17426596/437/1/012001

[48] J. I. González Hernández, R. Rebolo, J. Casares, Mon. Not. R. Astron. Soc., 000, 1-6 (2013). 